\title{
On nonlocal symmetries, nonlocal conservation laws and nonlocal transformations of evolution equations: Two linearisable hierarchies
}

\author{
Norbert Euler and Marianna Euler \\ Department of Mathematics, Luleå University of Technology \\ SE-971 87 Luleå, Sweden \\ Norbert.Euler@sm.luth.se; Marianna.Euler@sm.luth.se
}

\begin{abstract}
We discuss nonlocal symmetries and nonlocal conservation laws that follow from the systematic potentialisation of evolution equations. Those are the Lie point symmetries of the auxiliary systems, also known as potential symmetries. We define higherdegree potential symmetries which then lead to nonlocal conservation laws and nonlocal transformations for the equations. We demonstrate our approach and derive second degree potential symmetries for the Burgers' hierarchy and the Calogero-Degasperis-IbragimovShabat hierarchy.
\end{abstract}

\section{Introduction}

The concept of nonlocal symmetries of partial differential equations and its relations to local Lie point symmetries of its associated auxiliary systems was introduced by Bluman, Kumei and Reid [3] and are known as potential symmetries. We point out that more general type of potential symmetries were introduced earlier by Krasil'shchik and Vinogradov (see [13] and [20]).

In the present paper our starting point is based on potential symmetries as introduced in [3] and [2]. We define higher-degree nonlocal symmetries for evolution equations by introducing further auxiliary system by higher-degree potentialisations. This leads to nonlocal conservation laws for the given evolution equations and to nonlocal transformations between the evolution equations and its potentialised equations. We demonstrate our approach by considering the well-known Burgers' hierarchy and the so-called CalogeroDegasperis-Ibragimov-Shabat hierarchy ([5], [12], ([19], ([4]). Both of these hierarchies are known to be linearisable (see e.g. [4], [7] and [16]. See also [18] for a discussion on nonlocal symmetries of the Calogero-Degasperis-Ibragimov-Shabat equation). We show that the linearisations of the two hierarchies follow directly from their second potentialisations. An interesting and unexpected result of our investigation is that second-degree potential symmetries (as defined by Definition 2.1) exist only for the first members of both the Burgers' and the Calogero-Degasperis-Ibragimov-Shabat hierarchies.

On the notation: Throughout this paper $D_{a}[p]$ denotes the total derivative-operator of the dependent variable $p(a, b)$ with respect to the independent variable $a$, where subscripts 
of $p$ denote partial derivatives:

$$
D_{a}[p]:=\frac{\partial}{\partial a}+p_{a} \frac{\partial}{\partial p}+p_{a a} \frac{\partial}{\partial p_{a}}+p_{a b} \frac{\partial}{\partial p_{b}}+p_{3 a} \frac{\partial}{\partial p_{a a}}+\cdots .
$$

The formal inverse-operator of $D_{a}[p]$ is denoted by $D_{a}^{-1}$, such that

$$
D_{a}^{-1} \circ D_{a}[p] \varphi=D_{a}[p] \circ D_{a}^{-1} \varphi=\varphi .
$$

Moreover

$$
D_{a}^{n}[p] \varphi=D_{a}^{n-1}[p] \circ D_{a}[p] \varphi, \quad n \in \mathcal{N} .
$$

If the dependence of the operator $D_{a}[p]$ on $p$ is obvious, we write just $D_{a}$ instead of $D_{a}[p]$.

\section{Preliminaries and higher-degree potential symmetries}

Consider an $n$ th-order evolution equation of the general form

$$
u_{t}=F\left(x, u, u_{x}, u_{x x}, u_{3 x}, \ldots, u_{n x}\right) \text {. }
$$

Assume that (2.1) is a symmetry-integrable evolution equation [10, i.e. (2.1) admits a hereditary recursion operator $R[u]$ such that

$$
\left[L_{F}[u], R[u]\right]=D_{t}[u] R[u]
$$

where $L_{F}[u]$ is the linear operator

$$
L_{F}[u]:=\frac{\partial F}{\partial u}+\frac{\partial F}{\partial u_{x}} D_{x}+\frac{\partial F}{\partial u_{x x}} D_{x}^{2}+\cdots .
$$

Assume further that the hierarchy of symmetry-integrable evolution equations can be presented in the form

$$
u_{t}=R^{n}[u] u_{x}, \quad n \in \mathcal{N},
$$

such that (2.1) corresponds to the first member of the hierarchy (2.4) with $n=1$. The conserved current, $\Phi^{t}$, for (2.1) must satisfy the relation ([11], [1])

$$
\Lambda=\hat{E}[u] \Phi^{t},
$$

where $\Lambda$ denotes an integrating factor for (2.1), i.e.

$$
\hat{E}[u]\left(\Lambda u_{t}-\Lambda F\left(x, u, u_{x}, u_{x x}, \ldots u_{n x}\right)\right)=0 .
$$

Here $\hat{E}[u]$ is the Euler operator

$$
\hat{E}[u]:=\frac{\partial}{\partial u}-D_{t} \circ \frac{\partial}{\partial u_{t}}-D_{x} \circ \frac{\partial}{\partial u_{x}}+D_{x}^{2} \circ \frac{\partial}{\partial u_{x x}}-D_{x}^{3} \circ \frac{\partial}{\partial u_{3 x}}+\cdots .
$$


For the flux, $\Phi^{x}$, we state

Proposition 2.1: Let $\Lambda$ be an integrating factor for the evolution equations (2.1) and assume that the corresponding conserved current, $\Phi^{t}$, admits the dependence

$$
\Phi^{t}=\Phi^{t}\left(x, u, u_{x}, u_{x x}, u_{3 x}\right) .
$$

Then the flux, $\Phi^{x}$, for (2.1) is given by

$$
\begin{aligned}
\Phi^{x}= & -D_{x}^{-1}(\Lambda F)-\frac{\partial \Phi^{t}}{\partial u_{x}} F-\frac{\partial \Phi^{t}}{\partial u_{x x}} D_{x} F-\frac{\partial \Phi^{t}}{\partial u_{3 x}} D_{x}^{2} F \\
& +F D_{x}\left(\frac{\partial \Phi^{t}}{\partial u_{x x}}\right)-F D_{x}^{2}\left(\frac{\partial \Phi^{t}}{\partial u_{3 x}}\right)+\left(D_{x} F\right) D_{x}\left(\frac{\partial \Phi^{t}}{\partial u_{3 x}}\right) .
\end{aligned}
$$

The hierarchy (2.4) admits the same integrating factor, $\Lambda$, as the first member of the hierarchy (for $n=1$ ) and hence the same corresponding current, $\Phi^{t}$. The flux, $\Phi^{x}$, for the hierarchy, 2.4), for all $n \in \mathcal{N}$ then takes the fom

$$
\begin{aligned}
& \Phi^{x}\left(x, u, u_{x}, \ldots ; n\right)=-D_{x}^{-1}\left(\Lambda R^{n}[u] F\right)-\frac{\partial \Phi^{t}}{\partial u_{x}} R^{n}[u] F-\frac{\partial \Phi^{t}}{\partial u_{x x}} D_{x}\left(R^{n}[u] F\right) \\
& \quad-\frac{\partial \Phi^{t}}{\partial u_{3 x}} D_{x}^{2}\left(R^{n}[u] F\right)+\left(R^{n}[u] F\right) D_{x}\left(\frac{\partial \Phi^{t}}{\partial u_{x x}}\right)-\left(R^{n}[u] F\right) D_{x}^{2}\left(\frac{\partial \Phi^{t}}{\partial u_{3 x}}\right) \\
& \quad+D_{x}\left(R^{n}[u] F\right) D_{x}\left(\frac{\partial \Phi^{t}}{\partial u_{3 x}}\right),
\end{aligned}
$$

where we assume the dependence of $\Phi^{t}$ as stated in (2.8).

Remark: The proof of Proposition 2.1 is straightforward, namely by integrating the conservation law

$$
\left.\left(D_{t} \Phi^{t}+D_{x} \Phi^{x}\right)\right|_{u_{t}=R^{n}[u] u_{x}}=0
$$

of the hierarchy (2.4) with respect to $x$, that is

$$
\Phi^{x}=-\left.D_{x}^{-1}\left(D_{t} \Phi^{t}\right)\right|_{u_{t}=R^{n}[u] u_{x}} .
$$

Assume now that the evolution equation (2.1) admits a conserved current, $\Phi_{1}^{t}$, and flux, $\Phi_{1}^{x}$. Following [3] a first potential variable $v$ is then defined by the auxiliary system:

$$
\begin{aligned}
& v_{x}=\Phi_{1}^{t}\left(x, u, u_{x}, \ldots\right) \\
& v_{t}=-\Phi_{1}^{x}\left(x, u, u_{x}, \ldots\right) .
\end{aligned}
$$

We name system (2.12a) - (2.12b) the first auxiliary system of (2.1). Assume further that $(2.12 \mathrm{~b})$ can be expressed in terms of the first potential variable $v$, i.e. (2.12b) becomes by (2.12a) the first potential equation of the general form

$$
v_{t}=G\left(x, v_{x}, v_{x x}, \ldots, v_{n x}\right)
$$


which may again admit a conserved current, $\Phi_{2}^{t}$, and flux, $\Phi_{2}^{x}$. A further potential $w$ is then introduced for (2.13), and named the second potential for (2.1), by the second auxiliary system

$$
\begin{aligned}
& w_{x}=\Phi_{2}^{t}\left(x, v, v_{x}, \ldots\right) \\
& w_{t}=-\Phi_{2}^{x}\left(x, v, v_{x}, \ldots\right) .
\end{aligned}
$$

The corresponding potential equation for (2.13) is then obtained from (2.14a) and (2.14b), which we assume to have the general form

$$
w_{t}=H\left(x, w_{x}, w_{x x}, \ldots, w_{n x}\right) .
$$

We name (2.15) the second potential equation for (2.1).

We now introduce the following

Definition 2.1: The Lie point symmetry generators

$$
Z=\xi_{1}(x, t, u, v) \frac{\partial}{\partial x}+\xi_{2}(x, t, u, v) \frac{\partial}{\partial t}+\eta_{1}(x, t, u, v) \frac{\partial}{\partial u}+\eta_{2}(x, t, u, v) \frac{\partial}{\partial v}
$$

of the first auxiliary system (2.12a)-(2.12b) for (2.1), i.e.

$$
\begin{aligned}
& v_{x}=\Phi_{1}^{t}\left(x, u, u_{x}, \ldots\right) \\
& v_{t}=-\Phi_{1}^{x}\left(x, u, u_{x}, \ldots\right),
\end{aligned}
$$

are defined as the first-degree potential symmetries of (2.1) if the infinitesimals $\xi_{1}, \xi_{2}$ and $\eta_{1}$ depend essentially on the first potential variable $v$, that is

$$
\left(\frac{\partial \xi_{1}}{\partial v}\right)^{2}+\left(\frac{\partial \xi_{2}}{\partial v}\right)^{2}+\left(\frac{\partial \eta_{1}}{\partial v}\right)^{2} \neq 0
$$

The second-degree potential symmetries of (2.1) are defined by the Lie point symmetry generators of the combined first- and second-auxiliary systems (2.12a)-(2.12b) and (2.14a)(2.14b), that is the Lie point symmetry generators of the form

$$
\begin{aligned}
Z= & \xi_{1}(x, t, u, v, w) \frac{\partial}{\partial x}+\xi_{2}(x, t, u, v, w) \frac{\partial}{\partial t}+\eta_{1}(x, t, u, v, w) \frac{\partial}{\partial u} \\
& +\eta_{2}(x, t, u, v, w) \frac{\partial}{\partial v}+\eta_{3}(x, t, u, v, w) \frac{\partial}{\partial w}
\end{aligned}
$$

for the system

$$
\begin{aligned}
& v_{x}=\Phi_{1}^{t}\left(x, u, u_{x}, \ldots\right) \\
& v_{t}=-\Phi_{1}^{x}\left(x, u, u_{x}, \ldots\right) \\
& w_{x}=\Phi_{2}^{t}\left(x, v, v_{x}, \ldots\right) \\
& w_{t}=-\Phi_{2}^{x}\left(x, v, v_{x}, \ldots\right),
\end{aligned}
$$


where the infinitesimals $\xi_{1}, \xi_{2}, \eta_{1}$ and $\eta_{2}$ depend essentially on the second potential variable $w$, that is

$$
\left(\frac{\partial \xi_{1}}{\partial w}\right)^{2}+\left(\frac{\partial \xi_{2}}{\partial w}\right)^{2}+\left(\frac{\partial \eta_{1}}{\partial w}\right)^{2}+\left(\frac{\partial \eta_{2}}{\partial w}\right)^{2} \neq 0
$$

It should be clear that Definition 2.1 can easily be extended to $m$ th-degree potential symmetries.

\section{The Burgers' hierarchy}

Consider the Burgers' equation in the form

$$
u_{t}=u_{x x}+2 u u_{x}
$$

It is well known that (3.1) admits only one local integrating factor and one local conservation law (see e.g. [15]), where

$$
\Lambda=1, \quad \Phi_{1}^{t}=u, \quad \Phi_{1}^{x}=-\left(u_{x}+u^{2}\right) .
$$

Equation (3.1) admits the recursion operator [14]

$$
R[u]=D_{x}+u+u_{x} D_{x}^{-1} \circ 1
$$

and the Burgers' hierarchy then takes the form

$$
u_{t}=R^{n}[u] u_{x}, \quad n=1,2, \ldots
$$

We remark that a general class of linearisable second-order evolution equations and its recursion operators, for which the Burgers' hierarchy is a special case, was reported in 8 and [7].

\subsection{Nonlocal conservation laws and linearisation}

We prove the following

Proposition 3.1: The Burgers' hierarchy 3.4,

$$
u_{t}=R^{n}[u] u_{x}, \quad n=1,2, \ldots,
$$

with $R$ given by (3.3) admits the first potentialisation of the form

$$
v_{t}=P^{n}\left[v_{x}\right] v_{x}, \quad n=1,2, \ldots,
$$

where

$$
P\left[v_{x}\right]=D_{x}\left[v_{x}\right]+v_{x},
$$


and the second potentialisation

$$
w_{t}=w_{(n+1) x}, \quad n=1,2, \ldots,
$$

where

$$
\begin{aligned}
& v_{x}=u \\
& v_{t}=P^{n}[u] u, \quad n=1,2, \ldots, \\
& w_{x}=e^{v} \\
& w_{t}=D_{x}^{n}[v] e^{v}, \quad n=1,2, \ldots,
\end{aligned}
$$

and

$$
P[u]=D_{x}+u
$$

The corresponding nonlocal conserved current, $\Phi^{t}$, and flux, $\Phi^{x}$, for hierarchy (3.4) are

$$
\begin{aligned}
& \Phi^{t}=e^{\int u d x} \\
& \Phi^{x}=-D_{x}^{n}[u]\left(e^{\int u d x}\right), \quad n=1,2, \ldots
\end{aligned}
$$

and the linearising transformation that transforms (3.4) in (3.7) is

$$
w_{x}=e^{\int u d x} .
$$

Proof: By Proposition 2.1 the hierarchy (3.4) admits the following integrating factor, $\Lambda$, conserved current, $\Phi_{1}^{t}$, and flux, $\Phi_{1}^{x}$ :

$$
\begin{aligned}
& \Lambda=1 \\
& \Phi_{1}^{t}(u)=u \\
& \Phi_{1, n}^{x}=-D_{x}^{-1}\left(R^{n}[u] u_{x}\right), \quad n=1,2, \ldots,
\end{aligned}
$$

where $R$ is the recursion operator, (3.3). It is easy to verify that

$$
D_{x}^{-1}\left(R^{n}[u] u_{x}\right)=P^{n}[u] u, \quad n=1,2, \ldots,
$$

where $P$ is defined by (3.9). The first auxiliary system for the Burgers' hierarchy (3.4) is then defined in terms of a potential variable $v$ in the form (3.8a) $-(3.8 \mathrm{~b})$, i.e.

$$
\begin{aligned}
& v_{x}=u \\
& v_{t}=P^{n}[u] u, \quad n=1,2, \ldots,
\end{aligned}
$$

and the first potential hierarchy of the Burgers' hierarchy, (3.4), becomes (3.5), i.e.

$$
v_{t}=P^{n}\left[v_{x}\right] v_{x}, \quad n=1,2, \ldots .
$$


The first potential hierarchy, (3.5), admits the following integrating factor, $\Lambda$, conserved current, $\Phi^{t}$, and flux, $\Phi^{x}$ :

$$
\begin{aligned}
& \Lambda=e^{v} \\
& \Phi_{2}^{t}(v)=e^{v} \\
& \Phi_{2, n}^{x}=-D_{x}^{-1}\left(e^{v} P^{n}\left[v_{x}\right] v_{x}\right), \quad n=1,2, \ldots .
\end{aligned}
$$

By the relation

$$
D_{x}^{-1}\left(e^{v} P^{n}\left[v_{x}\right] v_{x}\right)=D_{x}^{n}[v] e^{v}, \quad n=1,2, \ldots,
$$

the second auxiliary system of the Burgers' hierarchy (3.4) is then defined in the form (3.8c) $-(3.8 \mathrm{~d})$, i.e.

$$
\begin{aligned}
& w_{x}=e^{v} \\
& w_{t}=D_{x}^{n}[v] e^{v}, \quad n=1,2, \ldots,
\end{aligned}
$$

so that the second potential hierarchy becomes

$$
w_{t}=D_{x}^{n}[w] w_{x} \equiv w_{(n+1) x}, \quad n=1,2, \ldots
$$

The nonlocal transformation (3.11) follows directly from (3.8a) and (3.8c), namely the well known Cole-Hopf transformation (see e.g. [15]). The nonlocal conserved current (3.10a) and flux (3.10b) follows directly by expressing (3.8c) $-(3.8 \mathrm{~d})$ in terms of the original variable $u$.

\subsection{Potential symmetries of the Burgers' hierarchy}

We now turn our attention to the symmetry properties of the auxiliary systems (3.8a)(3.8b) and the combined auxiliary system (3.8a - (3.8d). Firstly we discuss in detail the cases $n=1$ and $n=2$.

Case $n=1$ : The first auxiliary system (3.8a - (3.8b) of hierarchy (3.4) with $n=1$ is

$$
\begin{aligned}
& v_{x}=u \\
& v_{t}=u_{x}+u^{2}
\end{aligned}
$$

and the first potential equation has the form

$$
v_{t}=v_{x x}+v_{x}^{2}
$$

By Definition 2.1 the first-degree potential symmetries of (3.1) are the Lie point symmetries 
of (3.18a $)-(3.18 \mathrm{~b})$. We obtain

$$
\begin{aligned}
& Z_{1}=\frac{\partial}{\partial t}, \quad Z_{2}=\frac{\partial}{\partial x}, \quad Z_{3}=\frac{\partial}{\partial v} \\
& Z_{4}=x \frac{\partial}{\partial x}+2 t \frac{\partial}{\partial t}-u \frac{\partial}{\partial u}, \quad Z_{5}=2 t \frac{\partial}{\partial x}-\frac{\partial}{\partial u}-x \frac{\partial}{\partial v} \\
& Z_{6}=4 x t \frac{\partial}{\partial x}+4 t^{2} \frac{\partial}{\partial t}-2(x+2 t u) \frac{\partial}{\partial u}-\left(2 t+x^{2}\right) \frac{\partial}{\partial v} \\
& Z_{\infty}=e^{-v}\left(\frac{\partial f}{\partial x}-u f(x, t)\right) \frac{\partial}{\partial u}+f(x, t) e^{-v} \frac{\partial}{\partial v}, \quad \text { where } f_{t}-f_{x x}=0 .
\end{aligned}
$$

The first-degree potential symmetries (3.20a - (3.20d) were firstly obtained by Vino'gradov and Krasil'shchik [20]. The second auxiliary system (3.8c)-(3.8d) for hierarchy (3.4) with $n=1$ is

$$
\begin{aligned}
& w_{x}=e^{v} \\
& w_{t}=v_{x} e^{v}
\end{aligned}
$$

and the second potential equation for hierarchy (3.4) with $n=1$ has the form

$$
w_{t}=w_{x x}
$$

Following Definition 2.1 the second-degree potential symmetries of the Burgers hierarchy (3.4) for $n=1$ are the Lie point symmetries of the combined auxiliary systems (3.18a)(3.18b) and (3.21a $)-(3.21 \mathrm{~b})$, i.e. the Lie point symmetries of the system

$$
\begin{aligned}
& v_{x}=u \\
& v_{t}=u_{x}+u^{2} \\
& w_{x}=e^{v} \\
& w_{t}=v_{x} e^{v} .
\end{aligned}
$$

We obtain the following second-degree potential symmetries of (3.4) for $n=1$ :

$$
\begin{aligned}
& Z_{1}=\frac{\partial}{\partial t}, \quad Z_{2}=\frac{\partial}{\partial x} \\
& Z_{3}=x \frac{\partial}{\partial x}+2 t \frac{\partial}{\partial t}-u \frac{\partial}{\partial u}+w \frac{\partial}{\partial w}, \quad Z_{4}=w \frac{\partial}{\partial w}+\frac{\partial}{\partial v} \\
& Z_{5}=2 t \frac{\partial}{\partial x}-\left(2-u w e^{-v}\right) \frac{\partial}{\partial u}-\left(x+w e^{-v}\right) \frac{\partial}{\partial v}-x w \frac{\partial}{\partial w}
\end{aligned}
$$




$$
\begin{aligned}
Z_{6}= & 2 x t \frac{\partial}{\partial x}+2 t^{2} \frac{\partial}{\partial t}-\left(2 x+2 t u+w e^{-v}-x u w e^{-v}\right) \frac{\partial}{\partial u} \\
& -\left(3 t+\frac{1}{2} x^{2}+x w e^{-v}\right) \frac{\partial}{\partial v}-\left(t w+\frac{1}{2} x^{2} w\right) \frac{\partial}{\partial w} \\
Z_{\infty} & =e^{-v}\left(u \frac{\partial f}{\partial x}-\frac{\partial^{2} f}{\partial x^{2}}\right) \frac{\partial}{\partial u}-e^{-v} \frac{\partial f}{\partial x} \frac{\partial}{\partial v}-f(x, t) \frac{\partial}{\partial w}
\end{aligned}
$$

where $f_{t}-f_{x x}=0$.

Case $n=2$ : The first auxiliary system (3.8a $)-(3.8 \mathrm{~b})$ of hierarchy (3.4) with $n=2$ is

$$
\begin{aligned}
& v_{x}=u \\
& v_{t}=u_{x x}+3 u u_{x}+u^{3}
\end{aligned}
$$

and the first potential equation has the form

$$
v_{t}=v_{3 x}+3 v_{x} v_{x x}+v_{x}^{3}
$$

The first-degree potential symmetries of (3.1) are then

$$
\begin{aligned}
& Z_{1}=\frac{\partial}{\partial t}, \quad Z_{2}=\frac{\partial}{\partial x}, \quad Z_{3}=\frac{\partial}{\partial v} \\
& Z_{4}=x \frac{\partial}{\partial x}+3 t \frac{\partial}{\partial t}-u \frac{\partial}{\partial u} \\
& Z_{\infty}=e^{-v}\left(\frac{\partial f}{\partial x}-u f(x, t)\right) \frac{\partial}{\partial u}+f(x, t) e^{-v} \frac{\partial}{\partial v},
\end{aligned}
$$

where $f_{t}-f_{3 x}=0$.

The second auxiliary system (3.8c) $-(3.8 \mathrm{~d})$ for hierarchy (3.4) with $n=2$ is

$$
\begin{aligned}
& w_{x}=e^{v} \\
& w_{t}=v_{x x} e^{v}+v_{x}^{2} e^{v}
\end{aligned}
$$

and the second potential equation for hierarchy (3.4) with $n=2$ has the form

$$
w_{t}=w_{3 x} .
$$

The second-degree potential symmetries of the Burgers' hierarchy (3.4) for $n=2$ would follow from the Lie point symmetries of the combined auxiliary systems (3.18a - (3.18b) and (3.21a $)-(3.21 \mathrm{~b})$, i.e. the Lie point symmetries of the system

$$
\begin{aligned}
& v_{x}=u \\
& v_{t}=u_{x x}+3 u u_{x}+u^{3} \\
& w_{x}=e^{v} \\
& w_{t}=v_{x x} e^{v}+v_{x}^{2} e^{v} .
\end{aligned}
$$


We obtain the following Lie point symmetries of system (3.31a $-(3.31 \mathrm{~d})$ :

$$
\begin{aligned}
& Z_{1}=\frac{\partial}{\partial t}, \quad Z_{2}=\frac{\partial}{\partial x} \\
& Z_{3}=x \frac{\partial}{\partial x}+3 t \frac{\partial}{\partial t}-u \frac{\partial}{\partial u}+w \frac{\partial}{\partial w}, \quad Z_{4}=w \frac{\partial}{\partial w}+\frac{\partial}{\partial v} \\
& Z_{\infty}=e^{-v}\left(u \frac{\partial f}{\partial x}-\frac{\partial^{2} f}{\partial x^{2}}\right) \frac{\partial}{\partial u}-e^{-v} \frac{\partial f}{\partial x} \frac{\partial}{\partial v}-f(x, t) \frac{\partial}{\partial w}
\end{aligned}
$$

where $f_{t}-f_{3 x}=0$.

It is clear that the above Lie point symmetry generators are not potential symmetries of second degree for the third-order Burgers' equation (3.27). The same happens for the case $n=3$, i.e., second-degree potential symmetries for the Burgers' hierarchy appear only for the case $n=1$, namely the Burgers' equation (3.1).

From the above patterns in the symmetry generators we allow ourselves the following

Supposition 3.1: There exist no second-degree potential symmetries for the Burgers' hierarchy (3.4) for $n>1$ and the maximum set of first-degree potential symmetries for the hierarchy (3.4) for all natural numbers $n>1$, is given by the following Lie symmetry generators:

$$
\begin{aligned}
& Z_{1}=\frac{\partial}{\partial t}, \quad Z_{2}=\frac{\partial}{\partial x}, \quad Z_{3}=\frac{\partial}{\partial v} \\
& Z_{4}=x \frac{\partial}{\partial x}+(n+1) t \frac{\partial}{\partial t}-u \frac{\partial}{\partial u} \\
& Z_{\infty}=e^{-v}\left(\frac{\partial f}{\partial x}-u f(x, t)\right) \frac{\partial}{\partial u}+f(x, t) e^{-v} \frac{\partial}{\partial v},
\end{aligned}
$$

where $f_{t}-f_{(n+1) x}=0$.

\subsection{Reciprocal-Bäcklund transformations of the Burgers' hierarchy}

With the general expressions of the conserved current, (3.12b), and flux, (3.12c), together with the relation (3.13) for the the Burgers' hierarchy (3.4), we use the opportunity to transform the hierarchy by a reciprocal-Bäcklund transformation (see e.g. [17] and [6]) and hence present a transformed Burgers' hierarchy. The following two Propositions give the result for both the Burgers' hierarchy (3.4) and the potential Burgers' hierarchy (3.5): 
Proposition 3.2: Under the reciprocal-Bäcklund transformation

$$
R:\left\{\begin{array}{l}
d y(x, t)=\Phi_{1}^{t} d x-\Phi_{1, n}^{x} d t \\
d \tau(x, t)=d t \\
U(y, \tau)=u
\end{array}\right.
$$

with

$$
\Phi_{1}^{t}=u, \quad \Phi_{1, n}^{x}=-P^{n}[u] u
$$

the Burgers' hierarchy

$$
u_{t}=R^{n}[u] u_{x}, \quad \text { where } R[u]=D_{x}[u]+u+u_{x} D_{x}^{-1} \circ 1,
$$

transforms to the hierarchy

$$
U_{\tau}=\left\{U D_{y}[U]-U_{y}\right\}\left\{P^{n}[U] U\right\},
$$

where

$$
P[U]=U D_{y}[U]+U .
$$

Proposition 3.3: Under the reciprocal-Bäcklund transformation

$$
R:\left\{\begin{array}{l}
d y(x, t)=\Phi_{2}^{t} d x-\Phi_{2, n}^{x} d t \\
d \tau(x, t)=d t \\
V(y, \tau)=v
\end{array}\right.
$$

with

$$
\Phi_{2}^{t}=e^{v}, \quad \Phi_{2, n}^{x}=-D_{x}^{n}[v] e^{v},
$$

the potential Burgers' hierarchy

$$
v_{t}=P^{n}\left[v_{x}\right] v_{x}, \quad \text { where } P\left[v_{x}\right]=D_{x}\left[v_{x}\right]+v_{x},
$$

transforms to the hierarchy

$$
V_{\tau}=\left\{D_{y}[V]-V_{y}\right\}\left\{\left(e^{V} D_{y}[V]\right)^{n} e^{V}\right\} .
$$

We give some explicit examples of the equations (3.37) and (3.42):

Under the reciprocal-Bäcklund transformation (3.34) -(3.35) the Burgers' hierarchy (3.36) with $n=1$ transforms to

$$
U_{\tau}=U^{2} U_{y y}+U^{2} U_{y}
$$


and for $n=2$ we obtain

$$
U_{\tau}=U^{3} U_{3 y}+3 U^{3} U_{y y}+3 U^{2} U_{y} U_{y y}+3 U^{2} U_{y}^{2}+2 U^{3} U_{y}
$$

Under the reciprocal-Bäcklund transformation (3.39)-(3.40) the potential Burgers' hierarchy (3.41) with $n=1$ transforms to

$$
V_{\tau}=e^{2 V}\left(V_{y y}+V_{y}^{2}\right)
$$

and for $n=2$ we obtain

$$
V_{\tau}=e^{3 V}\left(V_{3 y}+6 V_{y} V_{y y}+4 V_{y}^{3}\right)
$$

\section{The Calogero-Degasperis-Ibragimov-Shabat hierarchy}

The third-order evolution equation

$$
u_{t}=u_{3 x}+3 u^{2} u_{x x}+9 u u_{x}^{2}+3 u^{4} u_{x}
$$

is known as the Calogero-Degasperis-Ibragimov-Shabat equation and is a well-known $C$ integrable evolution equation which can be linearised by a nonlocal transformation ([19], [4], [16]). In [16] we derived a second-order nonlocal recursion operator for (4.1), namely

$$
\begin{aligned}
R[u] & =D_{x}^{2}+2 u^{2} D_{x}+10 u u_{x}+u^{4} \\
& +2\left(u_{x x}+2 u^{2} u_{x}+2 u e^{-2 \int u^{2} d x} \int e^{2 \int u^{2} d x} u_{x}^{2} d x\right) D_{x}^{-1} \circ u \\
& -2 u e^{-2 \int u^{2} d x} D_{x}^{-1} \circ\left[\left(u_{x x}+2 u^{2} u_{x}\right) e^{2 \int u^{2} d x}+2 u \int e^{2 \int u^{2} d x} u_{x}^{2} d x\right]
\end{aligned}
$$

and also reported some nonlocal symmetries that follow from this recursion operator. In terms of the recursion operator (4.2) a local Calogero-Degasperis-Ibragimov-Shabat hierarchy of $C$-integrable evolution equations can be presented in the form

$$
u_{t}=R^{n}[u] u_{x}, \quad n=1,2, \ldots .
$$

Equation (4.1) then corresponds to (4.3) with $n=1$. For $n=2$ the second member of hierarchy (4.3) is

$$
\begin{aligned}
u_{t}= & u_{5 x}+5 u^{2} u_{4 x}+40 u u_{x} u_{3 x}+25 u u_{x x}^{2}+50 u_{x}^{2} u_{x x}+10 u^{4} u_{3 x} \\
& +120 u^{3} u_{x} u_{x x}+140 u^{2} u_{x}^{3}+10 u^{6} u_{x x}+70 u^{5} u_{x}^{2}+5 u^{8} u_{x} .
\end{aligned}
$$

We now investigate the nonlocal symmetry structure in the sense of its first- and seconddegree potential symmetries and obtain the corresponding nonlocal conservation laws. We show that the linearisations of (4.1) and (4.4) follow directly from the second potentialisation of (4.1) and (4.4), respectively. 


\subsection{Nonlocal conservation laws and linearisation of the Calogero-Degas- peris-Ibragimov-Shabat hierarchy}

The results for the first and second members of the hierarchy (4.3) are given by the following two propositions:

Proposition 4.1: The Calogero-Degasperis-Ibragimov-Shabat equation (4.1),

$$
u_{t}=u_{3 x}+3 u^{2} u_{x x}+9 u u_{x}^{2}+3 u^{4} u_{x}
$$

admits a first potentialisation of the form

$$
v_{t}=v_{3 x}-\frac{3}{4} \frac{v_{x x}^{2}}{v_{x}}+3 v_{x} v_{x x}+v_{x}^{3}
$$

and second potentialisation of the form

$$
w_{t}=w_{3 x}
$$

where

$$
\begin{aligned}
& v_{x}=u^{2} \\
& v_{t}=2 u u_{x x}-u_{x}^{2}+6 u^{3} u_{x}+u^{6} \\
& w_{x}=e^{v} v_{x}^{1 / 2} \\
& w_{t}=e^{v}\left(\frac{1}{2} v_{x}^{-1 / 2} v_{3 x}-\frac{1}{4} v_{x}^{-3 / 2} v_{x x}^{2}+2 v_{x}^{1 / 2} v_{x x}+v_{x}^{5 / 2}\right) .
\end{aligned}
$$

The corresponding nonlocal conserved current, $\Phi^{t}$, and flux, $\Phi^{x}$, are

$$
\begin{aligned}
& \Phi^{t}=u e^{\int u^{2} d x} \\
& \Phi^{x}=-\left(u_{x x}+4 u^{2} u_{x}+u^{5}\right) e^{\int u^{2} d x}
\end{aligned}
$$

and the linearising transformation that transforms (4.1) to (4.6) is

$$
w_{x}=u e^{\int u^{2} d x} .
$$

For the second member of the Calogero-Degasperis-Ibragimov-Shabat hierarchy we have

Proposition 4.2: The second Calogero-Degasperis-Ibragimov-Shabat equation 4.4),

$$
\begin{aligned}
u_{t}= & u_{5 x}+5 u^{2} u_{4 x}+40 u u_{x} u_{3 x}+25 u u_{x x}^{2}+50 u_{x}^{2} u_{x x}+10 u^{4} u_{3 x} \\
& +120 u^{3} u_{x} u_{x x}+140 u^{2} u_{x}^{3}+10 u^{6} u_{x x}+70 u^{5} u_{x}^{2}+5 u^{8} u_{x}
\end{aligned}
$$


admits a first potentialisation of the form

$$
\begin{aligned}
v_{t}= & v_{5 x}+5 v_{x} v_{4 x}-\frac{5}{2} v_{x}^{-1} v_{x x} v_{4 x}+10 v_{x}^{2} v_{3 x}+5 v_{x x} v_{3 x}+5 v_{x}^{-2} v_{x x}^{2} v_{3 x} \\
& -\frac{5}{4} v_{x}^{-1} v_{3 x}^{2}-\frac{35}{16} v_{x}^{-3} v_{x x}^{4}-\frac{5}{2} v_{x}^{-1} v_{x x}^{3}+\frac{25}{2} v_{x} v_{x x}^{2}+10 v_{x}^{3} v_{x x}+v_{x}^{5}
\end{aligned}
$$

and second potentialisation of the form

$$
w_{t}=w_{5 x}
$$

where

$$
\begin{aligned}
v_{x}= & u^{2} \\
v_{t}= & 2 u u_{4 x}-2 u_{x} u_{3 x}+u_{x x}^{2}+10 u^{3} u_{3 x}+50 u^{2} u_{x} u_{x x}+20 u^{5} u_{x x}+70 u^{4} u_{x}^{2} \\
& +20 u^{7} u_{x}+u^{10} \\
w_{x}= & e^{v} v_{x}^{1 / 2} \\
w_{t}= & e^{v}\left(\frac{1}{2} v_{x}^{-1 / 2} v_{5 x}-v_{x}^{-3 / 2} v_{x x} v_{4 x}+3 v_{x}^{1 / 2} v_{4 x}-\frac{3}{4} v_{x}^{-3 / 2} v_{3 x}^{2}+\frac{9}{4} v_{x}^{-5 / 2} v_{x x}^{2} v_{3 x}\right. \\
+ & \left.2 v_{x}^{-1 / 2} v_{x x} v_{3 x}+7 v_{x}^{3 / 2} v_{3 x}-\frac{15}{16} v_{x}^{-7 / 2} v_{x x}^{4}+\frac{15}{2} v_{x}^{1 / 2} v_{x x}^{2}+8 v_{x}^{5 / 2} v_{x x}+v_{x}^{9 / 2}\right) .
\end{aligned}
$$

The corresponding nonlocal conserved current, $\Phi^{t}$, and flux, $\Phi^{x}$, are

$$
\begin{aligned}
\Phi^{t}= & u e^{\int u^{2} d x} \\
\Phi^{x}= & -\left(u_{4 x}+26 u u_{x} u_{x x}+6 u^{2} u_{3 x}+8 u_{x}^{3}+44 u^{3} u_{x}^{2}\right. \\
& \left.+14 u^{4} u_{x x}+16 u^{6} u_{x}+u^{9}\right) e^{\int u^{2} d x}
\end{aligned}
$$

and the linearising transformation that transforms (4.4) to 4.11) is

$$
w_{x}=u e^{\int u^{2} d x} .
$$

In order to derive the auxiliary systems for the Calogero-Degasperis-Ibragimov-Shabat hierarchy, (4.3), we need the integrating factors of this hierarchy and the integrating factors of the corresponding potential hierarchy. For the first two members of the hierarchy the integrating factors are given by the following

Lemma 4.1: The third-order Calogero-Degasperis-Ibragimov-Shabat equation, 4.1), and the fifth-order Calogero-Degasperis-Ibragimov-Shabat equation, 4.4), admit only one integrating factor, $\Lambda$, namely

$$
\Lambda\left(x, u, u_{x}, \ldots\right)=u \text {. }
$$


For the first potentialisation of (4.1), namely for the third-order potential equation (4.5), the complete set of integrating factors of second-order are

$$
\Lambda\left(x, v, v_{x}, v_{x x}\right)=a(x) e^{v} v_{x}^{-3 / 2} v_{x x}+2 a(x) e^{v} v_{x}^{1 / 2}-2 e^{v} v_{x}^{-1 / 2} \frac{d a}{d x}
$$

where

$$
\frac{d^{3} a}{d x^{3}}=0
$$

and for the first potentialisation of (4.4), namely for the fifth-order potential equation (4.10), the complete set of integrating factors of second-order are

$$
\Lambda\left(x, v, v_{x}, v_{x x}\right)=a(x) e^{v} v_{x}^{-3 / 2} v_{x x}+2 a(x) e^{v} v_{x}^{1 / 2}-2 e^{v} v_{x}^{-1 / 2} \frac{d a}{d x}
$$

where

$$
\frac{d^{5} a}{d x^{5}}=0
$$

To prove Lemma 4.1 we just verify (2.6).

Lemma 4.1 tempts us to make the following

Supposition 4.1: All first potentialisations of the Calogero-Degasperis-Ibragimov-Shabat hierarchy, (4.3), for all $n \in \mathcal{N}$ admit the following complete set of integrating factors of second order:

$$
\Lambda\left(x, u, u_{x}, u_{x x} ; n\right)=a(x) e^{v} v_{x}^{-3 / 2} v_{x x}+2 a(x) e^{v} v_{x}^{1 / 2}-2 e^{v} v_{x}^{-1 / 2} \frac{d a}{d x},
$$

where

$$
\frac{d^{n} a}{d x^{n}}=0
$$

Remark on the proof of Proposition 4.1 and Proposition 4.2:

For the linearisations of (4.1) and (4.4) in (4.6) and (4.11), respectively, we make use of the integrating factor

$$
\Lambda=e^{v} v_{x}^{-3 / 4} v_{x x}+2 e^{v} v_{x}^{1 / 2}
$$

which corresponds to the case $a(x)=1$ in Lemma 4.1. If one uses instead the explicitly $x$-dependent integrating factors, the resulting linear equations also depend explicitly on $x$. 


\subsection{Potential symmetries of the Calogero-Degasperis-Ibragimov-Shabat hierarchy}

We now study the symmetry properties of the auxiliary systems for the Calogero-DegasperisIbragimov-Shabat hierarchy (4.3).

Case $n=1$ : The first-degree potential symmetries of the first member of the hierarchy (4.3), i.e. (4.1), are given by the Lie point symmetries of the first auxiliary system (4.7a)(4.7b). We obtain

$$
\begin{aligned}
& Z_{1}=\frac{\partial}{\partial t}, \quad Z_{2}=\frac{\partial}{\partial x}, \quad Z_{3}=\frac{\partial}{\partial v} \\
& Z_{4}=\frac{1}{3} x \frac{\partial}{\partial x}+t \frac{\partial}{\partial t}-\frac{1}{6} u \frac{\partial}{\partial u}, \quad Z_{5}=u e^{-2 v} \frac{\partial}{\partial u}-e^{-2 v} \frac{\partial}{\partial v} .
\end{aligned}
$$

The second-degree potential symmetries of the first member of the hierarchy (4.3) are given by the Lie point symmetries of the combined auxiliary system (4.7a)-(4.7d). We obtain

$$
\begin{aligned}
& Z_{1}=\frac{\partial}{\partial t}, \quad Z_{2}=\frac{\partial}{\partial x}, \quad Z_{3}=\frac{\partial}{\partial w} \\
& Z_{4}=\frac{1}{3} x \frac{\partial}{\partial x}+t \frac{\partial}{\partial t}-\frac{1}{6} u \frac{\partial}{\partial u}+\frac{1}{6} w \frac{\partial}{\partial w}, \quad Z_{5}=\frac{\partial}{\partial v}+w \frac{\partial}{\partial w} \\
& Z_{6}=\left(\frac{1}{2} e^{-v}-u w e^{-2 v}\right) \frac{\partial}{\partial u}+w e^{-2 v} \frac{\partial}{\partial v}+\frac{1}{2} x \frac{\partial}{\partial w} \\
& Z_{7}=u e^{-2 v} \frac{\partial}{\partial u}-e^{-2 v} \frac{\partial}{\partial v} .
\end{aligned}
$$

Case $n=2$ : The first-degree potential symmetries of the second member of the hierarchy (4.3), i.e. (4.4), are given by the Lie point symmetries of the first auxiliary system (4.12a) (4.12b). We obtain

$$
\begin{aligned}
& Z_{1}=\frac{\partial}{\partial t}, \quad Z_{2}=\frac{\partial}{\partial x}, \quad Z_{3}=\frac{\partial}{\partial v} \\
& Z_{4}=\frac{1}{5} x \frac{\partial}{\partial x}+t \frac{\partial}{\partial t}-\frac{1}{10} u \frac{\partial}{\partial u}, \quad Z_{5}=u e^{-2 v} \frac{\partial}{\partial u}-e^{-2 v} \frac{\partial}{\partial v} .
\end{aligned}
$$

The complete set of Lie point symmetries of the auxiliary system (4.12a $-(4.12 \mathrm{~d}$ ) are

$$
\begin{aligned}
& Z_{1}=\frac{\partial}{\partial t}, \quad Z_{2}=\frac{\partial}{\partial x}, \quad Z_{3}=\frac{\partial}{\partial w} \\
& Z_{4}=\frac{1}{5} x \frac{\partial}{\partial x}+t \frac{\partial}{\partial t}-\frac{1}{10} u \frac{\partial}{\partial u}+\frac{1}{10} w \frac{\partial}{\partial w}, \quad Z_{5}=\frac{\partial}{\partial v}+w \frac{\partial}{\partial w} \\
& Z_{6}=u e^{-2 v} \frac{\partial}{\partial u}-e^{-2 v} \frac{\partial}{\partial v} .
\end{aligned}
$$


We note that the second member of the hierarchy (4.4) does not admit second-degree potential symmetries.

We allow ourselves the following

Supposition 4.2: There exist no second-degree potential symmetries for the CalogeroDegasperis-Ibragimov-Shabat hierarchy, (4.3), for $n>1$ and the maximum set of firstdegree potential symmetries for the hierarchy (4.3) is given by the following Lie symmetry generators:

$$
\begin{aligned}
& Z_{1}=\frac{\partial}{\partial t}, \quad Z_{2}=\frac{\partial}{\partial x}, \quad Z_{3}=\frac{\partial}{\partial v} \\
& Z_{4}=\frac{1}{n} x \frac{\partial}{\partial x}+t \frac{\partial}{\partial t}-\frac{1}{2 n} u \frac{\partial}{\partial u}, \quad Z_{5}=u e^{-2 v} \frac{\partial}{\partial u}-e^{-2 v} \frac{\partial}{\partial v},
\end{aligned}
$$

for all natural numbers $n>1$.

\section{Concluding remarks}

We have introduced second-degree potential symmetries in Definition 2.1 and studied the Burgers' hierarchy and the Calogero-Degasperis-Ibragimov-Shabat hierarchy. We obtained second-degree potential symmetries only for the first members of the hierarchies. Nonlocal conservation laws and nonlocal transformations which linearise the hierarchies were obtained through the second potentialisations. It would be interesting to investigate higher-degree potential symmetries further for other symmetry-integrable hierarchies and linearisable hierarchies as well as for systems of evolution equations. Preliminary calculations show that systematic potentialisation of some symmetry-integrable equations, such as the Krichever-Novikov equation, lead to interesting auto-Bäcklund transformations for the equations. A complete description of the potentialisation for a class of KrichiverNovikov equations and its connection with higher-degree potential symmetries is currently in preparation [9] and is planned for publication as a follow-up paper.

\section{Acknowledgement}

ME acknowledges the financial support provided by the LTU grant nr. 2557-05.

\section{References}

[1] Anco S and Bluman G W, Direct construction method for conservation laws of partial differential equations. II. General treatment European J. Appl. Math. 13 (2002), 567585 .

[2] Bluman G W and Kumei S, Symmetries and Differential Equations, Springer - New York, 1989.

[3] Bluman G W, Kumei S and Reid G J, New classes of symmetries of partial differential equations, J. Math. Phys. 29 (1988), 806-811. 
[4] Calogero F, The evolution partial differential equation $u_{t}=u_{x x x}+3\left(u_{x x} u^{2}+3 u_{x}^{2} u\right)+$ $3 u_{x} u^{4}$, J. Math. Phys. 28 (1987), 538-555.

[5] Calogero F and Degasperis A, Reduction technique for matrix nonlinear evolution equations solvable by the spectral transform, J. Math. Phys. 22 (1981), 23-31.

[6] Euler M, Euler N and Lundberg S, On reciprocal-Bäcklund transformations of autonomous evolution equations, Submitted, 2008.

[7] Euler M, Euler N and Petersson N, Linearizable hierarchies of evolution equations in $(1+1)$ dimensions Stud. in Appl. Math. 111 (2003) 315-337.

[8] Euler N and Euler M, A tree of linearisable second-order evolution equations by generalised hodograph transformations J. Nonlinear Math. Phys. 8 (2001) 342-362.

[9] Euler N and Euler M, Auto-Bäcklund transformations for Krichiver-Novikov type equations by potentialisations. In preparation.

[10] Fokas A S, Symmetries and integrability Stud. Appl. Math. 77 (1987), 253-299.

[11] Fokas A S and Fuchssteiner B, On the structure of symplectic operators and hereditary symmetries Lett. Nuovo Cimento (2) 28 (1980), 299-303.

[12] Ibragimov N H and Shabat A B, Infinite Lie-Bäcklund algebras, Funct. Anal. Appl. 14 (1981), 313-315.

[13] Krasil'shchik I S and Vinogradov A M, Nonlocal symmetries and the theory of coverings: an addendum to A M Vinogradov's "Local symmetries and conservation laws", Acta Appl. Math. 2 (1984), 79-96.

[14] Oevel W, Rekursionsmechanismen für Symmetrien und Erhaltungssätze in integrablen Systemen, PhD thesis, University of Paderborn, 1984.

[15] Olver P J, Applications of Lie Groups to Differential Equations, Springer - New York, 1986.

[16] Petersson N, Euler N and Euler M, Recursion operators for a class of integrable third-order evolution equations, Stud. Appl. Math. 112 (2004), 201-225.

[17] Rogers C and Shadwick W F, Bäcklund Transformations and Their Applications, Academic Press, New York, 1982.

[18] Sergyeyev A and Sanders J A, A remark on nonlocal symmetries for the CalogeroDegasperis-Ibragimov-Shabat equation, J. Nonlinear Math. Phys. 10 (2003), 78-85.

[19] Sokolov V V and Shabat A B, Classification of integrable evolution equations, Math. Phys. Rev. 4 (1984), 221-280.

[20] Vinogradov A M and Krasil'shchik I S, On the theory of nonlocal symmetries of nonlinear partial differential equations, Sov. Math. Dokl. 29 (1984) 337-341. 